\title{
Replacing the automatic gain control loop in a mobile, digital TV broadcast receiver by a software based solution
}

\author{
diploma thesis
}

Patrick Boettcher

Technische Fachhochschule Wildau

Fachbereich Betriebswirtschaft/Wirtschaftsinformatik

\author{
Date: \\ 09.03.2008 \\ Erstbetreuer: \\ Prof. Dr. Christian Müller \\ Zweitbetreuer: \\ Prof. Dr. Bernd Eylert
}


A part of this diploma thesis is not available until April 2010, because it is protected by a lock flag. The complete work can and will be made available by that time.

The parts affected are

- Chapter 4,

- Chapter 5,

- Appendix C and

- Appendix D.

If by that time you cannot find the complete work anywhere, please contact the author. 


\section{Danksagung}

An dieser Stelle möchte ich all jenen danken, die durch ihre fachliche und persönliche Unterstützung zum Gelingen dieser Diplomarbeit beigetragen haben.

Besonderer Dank gebührt meiner Lebenspartnerin Ariane und meinen Eltern, die mir dieses Studium durch ihre Unterstützung ermöglicht haben und mir fortwährend Vorbild und Ansporn waren.

Weiterhin bedanke ich mich bei Professor Dr. Christian Müller und Professor Dr. Bernd Eylert für die Betreuung dieser Diplomarbeit.

Großer Dank gilt ebenfalls meinen Kollegen bei DiBcom S.A., die mir die Möglichkeit gaben, diese Arbeit zu verfassen und mich technich sehr stark unterstützten.

Vor allem möchte ich mich in diesem Zusammenhang bei Jean-Philippe Sibers bedanken, der mir immer mit einer Inspriration zur Seite stand. Gleiches gilt für das „Physical Layer Software Team“: Luc Banda, Frédéric Tarral und Vincent Recrosio.

\section{Acknowledgment}

I want to use this opportunity to thank everyone who supported me personally and professionally to create this diploma thesis.

Special thanks appertain to my partner Ariane and my parents, who supported me during my studies and who continuously guided and motivated me.

Also I want to thank Professor Dr. Christian Müller and Professor Dr. Bernd Eylert for mentoring this diploma thesis.

A big thanks is going to all my colleagues at DiBcom S.A., who gave me the possibility to create this work and who supported me very strongly on a technical base.

In that context especially to Jean-Philippe Sibers who always had an inspiration for me. The same applies for the "Physical Layer Software Team": Luc Banda, Frédéric Tarral and Vincent Recrosio. 


\section{Abstract}

The power level (the amplitude) of an electro-magnetic signal wave suffers from attenuation the greater the distance between the transmitter and the receiver is. The receiver of that signal therefore has components which try to amplify the signal so that it can be processed optimally by a processor.

In a mobile or portable environment the signal power level can vary strongly, because the position of the receiver to the transmitter is not fixed. In order to compensate that movement a control loop exists, which dynamically is adapting the front-end to the right level.

This work describes a new, software-based way to handle the signal level control loop (formerly automatic gain control) in a digital TV receiver.

Starting with a very basic introduction into digital communications, including the description of the traditional front-end architecture, followed by a detailed description of the new method. Finally some conclusions of this new method are made which are giving an idea about how in the future it might be possible to reach better receiving performances using this mechanism. 


\section{Table of contents}

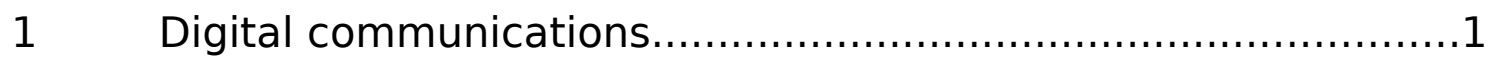

1.1 Digital communication model......................................... 1

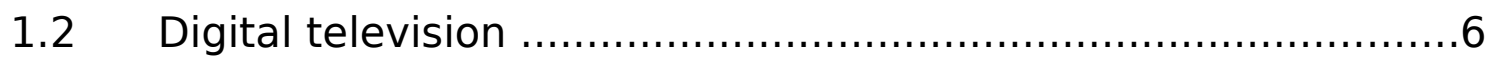

1.3 Digital TV broadcasting system......................................

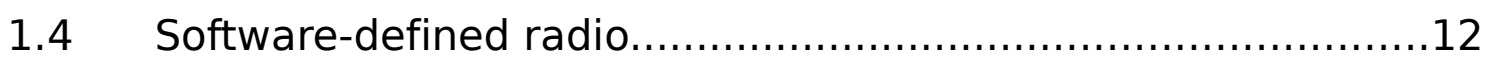

$2 \quad$ Digital TV receiver front-ends........................................13

$2.1 \quad$ Radio Frequency Tuner................................................13

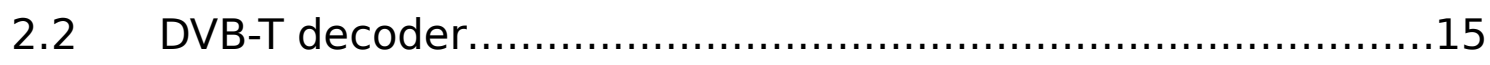

2.3 Interaction of decoder and tuner..................................16

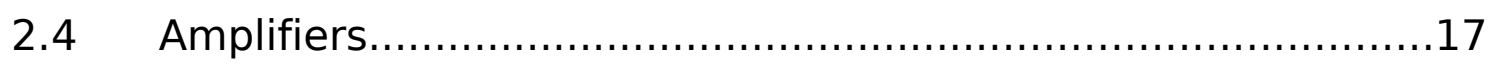

$2.5 \quad$ Analog to digital conversion......................................... 19

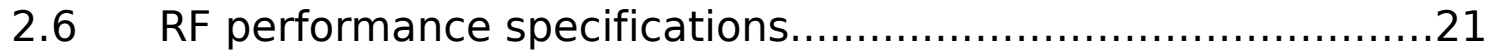

3 DiBcom front-end architecture.....................................24

$3.1 \quad$ Historical development at DiBcom....................................24

3.2 Traditional Automatic Gain Control...................................27

3.3 Next generation front-end architecture..............................30

$4 \quad$ Implementation of a software-based gain control.................32

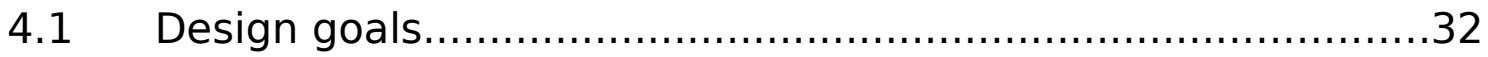

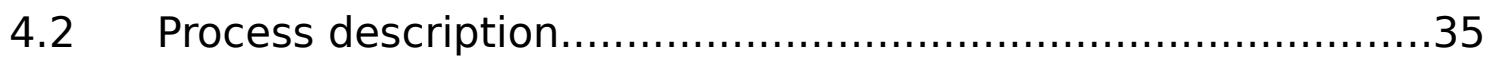

$4.3 \quad$ Comments to the source code..........................................43

4.4 Qualification................................................................ 48

$5 \quad$ Derived improvements, ideas and solutions.......................52

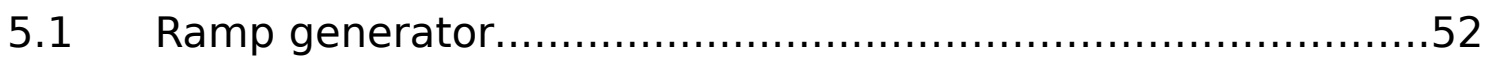

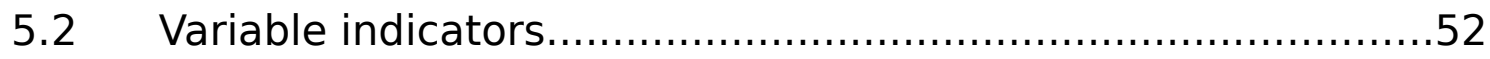

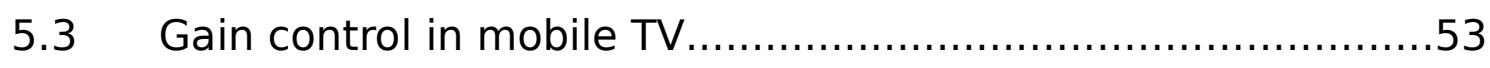

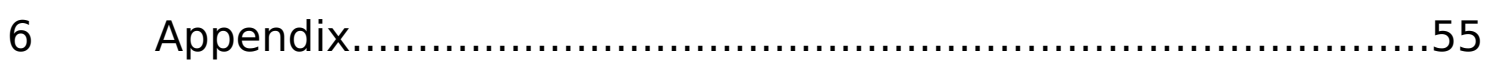




\section{Digital communications}

Exchanging information is one of the basic principle of individuals living in a society. The mutual exchange of information is called communication. The ways of communication are very versatile. In the human communication information is exchanged in the form of speech, sounds, images or text. One or more appropriate medias are selected by an individual who wants to transfer information to another one. The constraints to select the right transmission media are extensive as well. The right choice depends on the content and meaning of the information, the distance of the communicating entities and the environment.

Using digital methods to code information was and is still developing very fast during the last years. The efficiency and the much improved usage of the available bandwidth of the transmission spectrum are based on more and more complex and powerful technologies and, because of the latter, now usable algorithms.

The following sections are introducing the reader to the subject of this work.

\subsection{Digital communication model}

To introduce the important terms and to show the elements of a digital communication system a model was created. Fig. 1.1 is a representation chosen by [DIGNT02] and [DIGCOM01].

The source information might be either an analog signal, such as an audio or video signal, or a digital signal, which is discrete in time and has a finite number of symbols or characters. In a digital communication system, the source messages are coded in a binary form. The correct mechanisms have to be chosen to convert the information efficiently and to reduce redundancy. This process is called source encoding or data compression. The resulting information sequence is passed to the channel encoder, which adds redundancy to the sequence. This redundancy is used to help the receiver to recover from the effects of noise addition and interferences encountered in the transmission of the signal through the channel. 


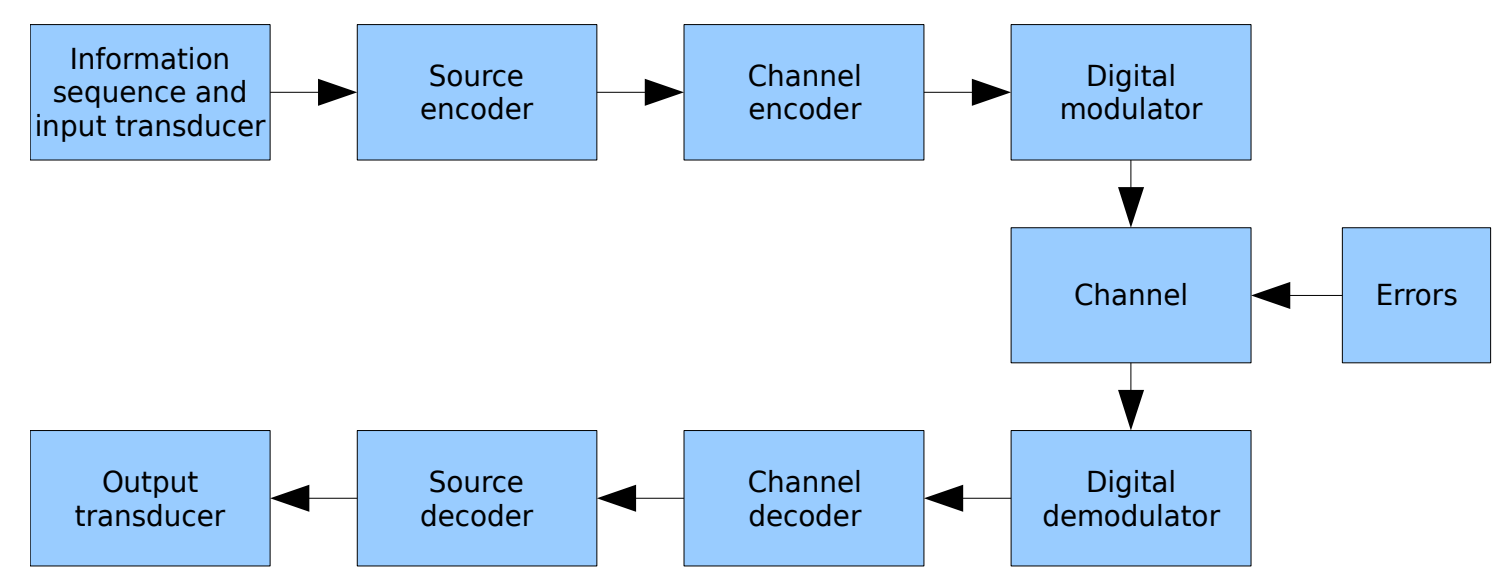

Fig. 1.1: Basic elements of a digital communication system

The interface between channel encoder and transmission channel is the digital modulator. The primary work of the modulator is to map the binary information sequence into signal waveforms.

[DIGNT02] is combining the channel encoder and digital modulator in one element, the transmission system. The transmission system is realizing the amplification, modulation, filtering and multiplexing of the electrical signal. Additionally it has to make sure, that the signal keeps its properties to be transmitted optimally over the transmission/communication channel. Is the channel optical an additional electro-optical conversion is taking place.

The transmission channel parameterized itself mainly through its physical media, which is for example a cable or the air for electrical signals, fiber cable for optical signals. The channel is the most sensitive part, simply because it is the most difficult one to protect against external negative impacts. It is thus affected by interferences and distortion noises, in general signal impairments. The signal may be corrupted randomly by a variety of possible mechanisms, such as additive thermal noise, man-made noise, for example automobile ignition noise.

The receiver is trying to undo all steps done at the transmitter. It is even trying to understand and to revert the signal impairments of the channel. At the receiving end of such a system, the digital demodulator processes the corrupted waveform and reduces it to a sequence of numbers that are an estimation of the transmitted data symbols. [DIGNT02] is describing the demodulator as the signal receiver. The mechanisms used to reproduce the original signal are amplification, filtering, equalization, demodulation, demultiplexing. 
The channel decoder is trying to recover the original information by using the redundant data added to the channel by the encoder. The goal is to get a reproduced information sequence.

Was the original information a analog structure, the source decoder will reconstruct the original signal. This can only be an approximation, because of channel decoding error and/or possible distortions introduced by the source encoder (lossy data compression).

The development of more and more efficient, cheaper and smaller (digital) technology allows to add digital processing methods at any step of a communication system.

It is necessary to distinguish between information which is digitally represented, digital signals and signals, which are processed digitally (by a processor).

Once the term digital is involved, the communication system will have at least one analog-to-digital converter ( $\mathrm{ADC}$ ) and a digital-to-analog converter (DAC). Section 2.5 on page 19 will describe the problematics of Analog-ToDigital conversion in detail.

\subsubsection{Digital video as an example for digital information}

The sources of video signals (for example a camera) produce analog signals, which are in an unlimited range of values, continuously progressing in time domain. These signals are discretely sampled, quantized and coded (see 2.5) by an analog-to-digital converter.

The digitalized analog TV of the year 2000 would use a net bit rate of around $166 \mathrm{MBit} / \mathrm{s}$. If this data rate would be transmitted without being compressed, more bandwidth would be required than for the present analog procedure. With the aid of an irrelevance reduction which takes into account the contents, higher reduction factors can be achieved without any subjective quality loss. This decreases the bandwidth far below than what is need for analog TV transmissions. How these mechanisms are working in detail is out of the scope of this work. Further readings about the principles can be found in [DVB05] on page 59 . 
With MPEG-2 video compression method a bandwidth of $6 \mathrm{MBit} / \mathrm{s}$ is sufficient to keep the quality on a standard TV set at least the same ${ }^{1}$.

For example in France one DVB-T RF channel carries 6 standard definition TV channels in the same frequency bandwidth as it was previously used by one analog TV channel.

\subsubsection{Signals, frequency and waves}

As mentioned in 1.1 in most communication systems the transmission channel is based on electrical signals. In electrical engineering a signal is a quantity which is varying in time or in space. ([WSIGNAL])

For example varying the voltage of an electrical signal. An electrical, modulated signal has the following representations: amplitude, frequency, phase. It is possible to observe a signal in time-domain and frequencydomain. Often a signal in one representation can be useful to acquire information whereas the same signal does not mean anything to the viewer in the other domain.

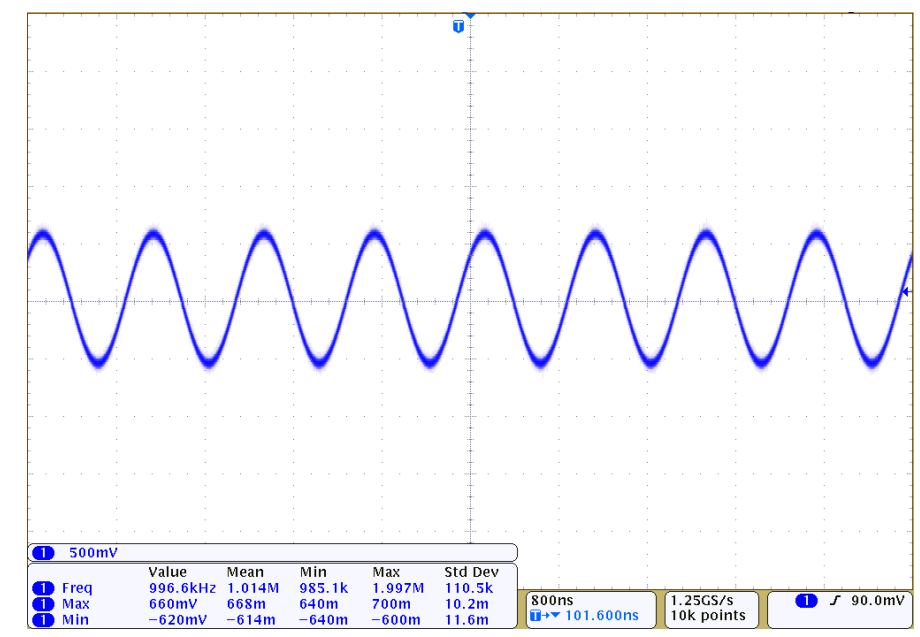

Fig. 1.2: A $1 \mathrm{MHz}$ sine wave in time-domain representation

Fig. 1.2 shows a sinusoidal signal at with a frequency of $1 \mathrm{MHz}$. At one point in this representation it is possible to observe the phase and the amplitude of the signal. Without the help of a measurement unit it is not very easy to the see frequency in that form. The very same signal observed with a

1 Most of the time the quality is better than that of analog TV because the transmission channel impairments in analog communication have a direct impact on the quality. 
frequency spectrum analyzer, and thus represented in the frequency domain, can be seen in Fig. 1.3.

In the frequency spectrum, which is the name used when showing a signal in frequency domain, the amplitude and frequency of the signal can be seen.

The mathematical method to transform a signal from time into the frequency domain and back is based on the Fourier analysis. With the help of the discrete Fourier transformation (DFT) a finite number of samples of a signal in time domain can be converted into frequency domain. In practice there are algorithms such as the Fast-Fourier-Transformation (FFT) which allow a very efficient conversion of signal data between the two representation forms. The discussion about Fourier analysis are described very well for example in [KNM07] and do not need to be repeated here. Appendix $B$ shows more examples of signals in different domains.

Both signal representations will be used later to describe some mechanisms and problems.

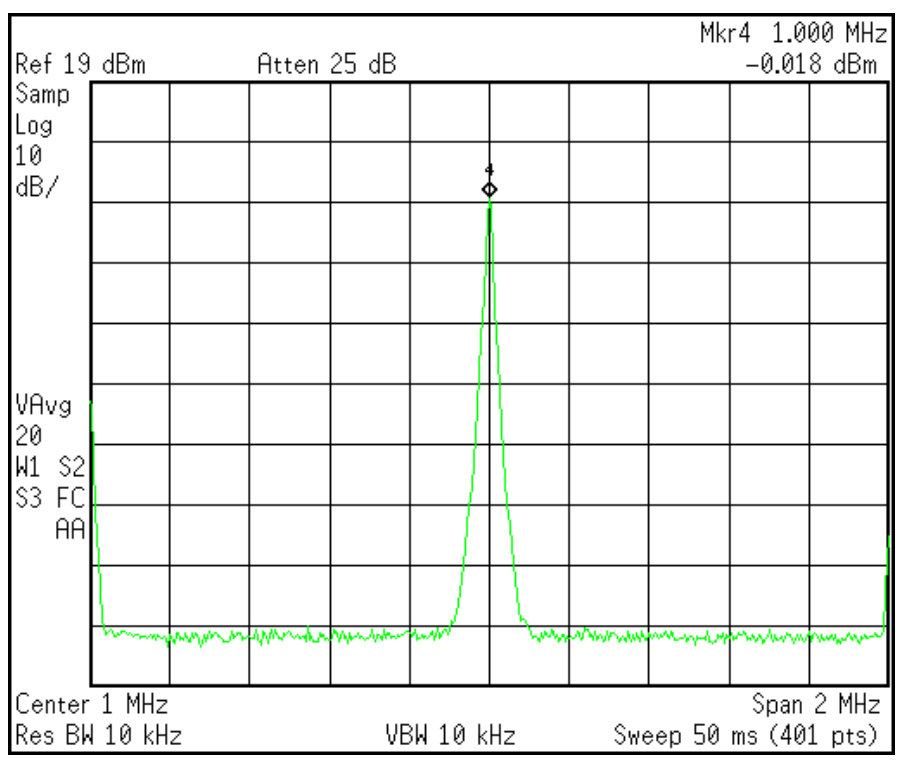

Fig. 1.3: A $1 \mathrm{MHz}$ sine wave in frequency-domain representation

\subsubsection{Digital signal processing}

Digital signal processing is the general term to process a signal using digital, 
numerical methods. Digital signal processors (DSP) can process any kind of signal - analog and digital. Most of the time the input of the DSP is an analog signal which was recorded and converted into a discrete signal by an Analog-to-Digital-Converter.

\subsection{Digital television}

Digital television is a form of digital communication using the broadcast principle.

There are several principles and directions to transmit information. In this work the focus is on broadband broadcast transmissions. Broadcasting is the uni-directional transmission of information to many receivers from one transmitter without having a feedback to the transmission source. The audience of broadcasting services is usually very big.

The term digital television "[..] refers to the source coding of audio, data and video signals, the channel coding and the methods for the transport of those digital signals via all kinds of transmission medias [..]" ([DVB05], p. vii).

World wide the development of digital television has started in the beginning of the 1990s.

\subsubsection{Europe (DVB)}

In Europe the DVB Project was formed 1992 to develop digital TV standard not only for Europe, but also for the rest of the world. The members of that project are either content provider, hardware manufacturers, network operators or regulatory authorities from more that 30 countries all over the world. As the coding for audio and video was already developed and to be compatible with existing decoding technology the project chose the MPEG-2 standards at the lowest application layer ${ }^{2}$.

The first complete system specification was the DVB-S recommendation for satellite transmissions in November 1993, which became a standard in

2 MPEG-2 audio and video standards and meta-information principles have been developed by the Moving Pictures Experts Group, independently of any digital TV standardization organization. It reports directly to ISO/IEC. 
1994. Still in 1994 the DVB-C specification followed, which describes the distribution digital TV over cable. In 1995 the DTT specification (digital terrestrial TV) DVB-T was adopted. In 1998 the first DVB-T network became available in the United Kingdom. ([DVB05], p. 6ff, p. 237)

\subsubsection{USA}

In the USA 1995 the Advanced Television Systems Committee (ATSC) approved the ATSC Digital Television Standard (A/53). In this standard the source coding is MPEG2 video and audio, the modulation used is vestigialsideband (VSB) modulation. ${ }^{3}$ Some broadcasters decided to use a quadrature amplitude modulation (QAM) which is very similar to the DVB-C. DirecTV/USSB/DSS is the name of the digital TV system very similar to DVB$S$ used for satellite transmission in the USA. ([DVB05] p. 5f)

\subsubsection{Japan}

In Japan, work on the development of digital television officially started in 1994. Before that, many Japanese companies have been involved in the DVB project, which is the reason for having some similarities between the specifications in the beginning. Since 1996, DVB-C was used for cable transmissions. Later the "Association of Radio Industries and Businesses" (ARIB) designated the system as Integrated Services Digital Broadcasting (ISDB). It includes ISDB-S (satellite), ISDB-C (cable) and ISDB-T (terrestrial). ([DVB05] p. 8f)

\subsubsection{Mobile TV}

Since 1981, Digital Audio Broadcasting (DAB) is developed. The idea of the development of DAB was in the first place to replace analog FM radio with this digital radio standard. In 1988 the first $D A B$ transmissions were made in Germany. DAB was the first standard based on orthogonal frequency division multiplexing (OFDM) modulation.

The acceptance of the audience of this digital radio standard was not very

3 Modulation of a single high-frequency carrier, 8-stages in terrestrial, 16-stages in cable transmissions 
high - most due to poor audio quality compared to FM radio.

A revival of the DAB standard has taken place in South Korea when the industry searched for a mobile digital TV standard. DAB was further developed to be able to carry MPEG4 coded video and other multimedia data. This extension is called DMB. ([WDAB])

The last years of digital TV development are more and more going to a portable or mobile reception.

The DVB project has developed DVB-H as an extension to DVB-T. Mechanisms like time-sliced broadcasting ${ }^{4}$ shall lower the power consumption of a battery-driven mobile receiver. Improved forward error correction shall improved the receiver sensitivity. In 2006 Italy has started DVB-H transmission, several other countries will follow in 2008; trails have been active in Germany, France and other places.

The latest development of the DVB Project for mobile digital TV is DVB-SH. In a hybrid broadcasted channel (satellite and terrestrial) the coverage of a big areas as well as in-house reception is tried to be ensured. ${ }^{5}$

The 3rd Generation Partnership Project (3GPP) has developed a broadcasting service called Multimedia Broadcast Multicast Service (MBMS) which can be deployed using existing GSM or UMTS physical layer technologies. Besides that the UMTS network is already able to deliver requested audio/video streams to the user of a cell phone in acceptable quality. It could be said, that this technology was enabling the first mobile TV service and that recent broadcast developments are basing their success on that idea.

3GPP publishes its standard as documents on the 3GPP website ${ }^{6}$. The standards produced by the DVB group be downloaded directly, free of charge, from the ETSI website ${ }^{7}$.

4 Highly compressed video data is transmitted in bursts, so that the most power consuming parts are not powered all the time. The received data is stored in memory. The bursts are timed in a way that a continuous stream is possible.

5 “DVB approves DVB-SH specification" - press release http://www.dvb.org/news_events/news/dvb_approves_dvbsh_specif/index.xml

6 http://www.3gpp.org/ftp/Specs/

7 http://www.etsi.org/WebSite/Standards/StandardsDownload.aspx 


\subsubsection{Overview}

The following table gives an overview of the digital broadcast TV standards and some of their parameters used world-wide.

\begin{tabular}{|c|c|c|}
\hline Standard & $\begin{array}{l}\text { Region, country } \\
\text { (selection) }\end{array}$ & Comment \\
\hline DVB-T & $\begin{array}{l}\text { Europe, Australia, South } \\
\text { Africa, Russia }\end{array}$ & $\begin{array}{l}\text { Terrestrial broadcast as a } \\
\text { replacement for analog TV }\end{array}$ \\
\hline DVB-S & Europe, USA (DSS), Japan, & $\begin{array}{l}\text { First digital TV broadcast in } \\
\text { Europe }\end{array}$ \\
\hline DVB-C & Europe, USA (QAM) & \\
\hline ATSC-T & USA, Canada, South Korea & Terrestrial broadcast \\
\hline DVB-H & $\begin{array}{l}\text { Europe (started 2006, still } \\
\text { developing) }\end{array}$ & $\begin{array}{l}\text { Extension of DVB-T for mobile } \\
\text { receivers (handhelds) }\end{array}$ \\
\hline $\mathrm{DAB} / \mathrm{T}-\mathrm{DMB}$ & $\begin{array}{l}\text { South Korea, studies in } \\
\text { Germany }\end{array}$ & Mobile TV alternative \\
\hline MBMS & Trials ongoing in the UK & $\begin{array}{l}\text { Broadcast-extension using } \\
\text { UMTS physical layer. }\end{array}$ \\
\hline
\end{tabular}

Table 1.1: Short overview of digital TV broadcast standards all over the world

\subsection{Digital TV broadcasting system}

Fig. 1.4 shows a block diagram of the transmitter and the opposite side, the receiver. In such a system not only video and audio data can be transferred. In fact any kind of digitalized data can be used. In practice the most common additional data added to the video and audio streams is the electronic program guides or electronic service guides which carry information about the available video and audio streams and other descriptions.

Different content providers are creating the content which is multiplexed into one single data stream. As of today every used Digital TV system is using MPEG-2 transport stream as the lowest application layer.

Besides the actual meaning of the information the communication system has to ensure the integrity and the correctness of the information transported at the receiver. As already mentioned the channel encoder adds 
redundancy to the source-coded digital signal to let the receiver correct errors. The addition of this redundancy increases the volume of the data to be transmitted.

In the transmission channel errors are causing the invalidation of one or more bits. A ' 1 ' becomes a ' 0 ' and vice versa. Evaluating and removing the

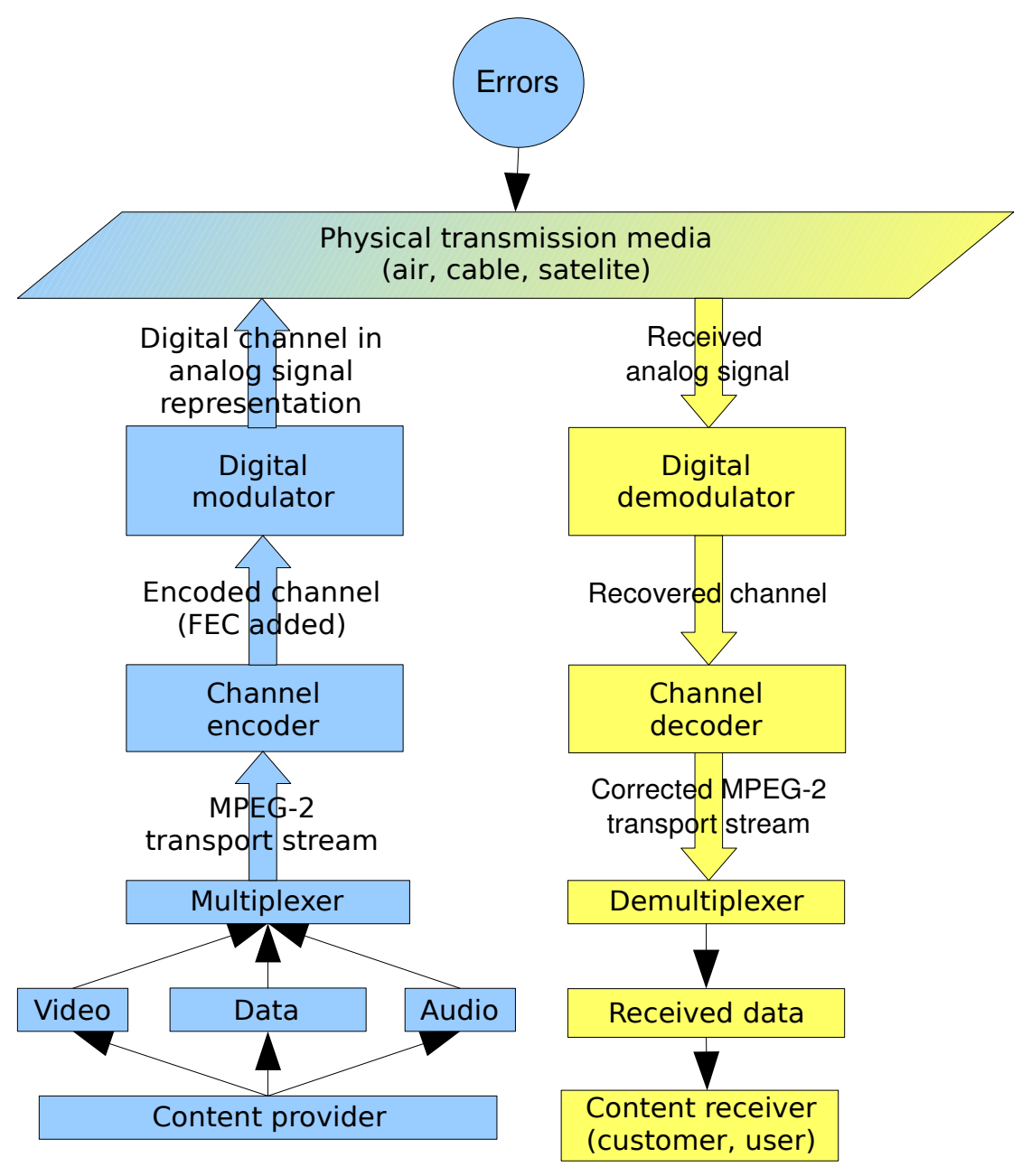

Fig. 1.4: Block diagram of a digital TV broadcasting system

redundancy in the receiver is the task of the channel decoder. It hereby tries to correct possibly occurred errors or at least provide the information whether a part of the data is erroneous or not.

With the knowledge of the types of errors in the channel, the right coding mechanism has to be chosen.

The channel decoder can sometimes report the bit-error-rate (BER). This rate gives the proportion of erroneously decoded bits to the total number of bits received. For DVB-T a bit error rate of $2 * 10^{-4}$ is marking the "Quasi-ErrorFree"-point (QEF) ("less than one uncorrected error event per hour"). 
([MBRAI07], p. 20)

Accordingly the packet error rate gives the rate of erroneously MPEG-2 transport stream packets received to the total number of packets received or expected to receive.

Interleaving ${ }^{8}$ the data in time helps the receiver to recover from long or bursted errors. The interleaver does not supply additional error correction codes - it initiates a rearrangement of the symbols ${ }^{9}$. Like that two consecutive symbols are not transmitted in that exact same order. ([DVB05], p. 111ff, p. 141f)

Some signal impairments are only impacting the signal for a short time but are repetitive (impulsive noise), some are having a constant impact (additive white Gaussian noise, AWGN). In a wireless environment reception echoes are faced and sometimes even wanted ${ }^{10}$. Is the delay between echoes too big, they are interfering each other. Is the receiver moving, several effects are causing so called fading which has - depending on the velocity of the receiver - a dramatic impact on the signal (inter-carrierinterference, inter-symbol-interference). This is just an extract of possible interferences a signal can encounter. Describing all of them in detail would exceed the scope of the work. For example [DIGNT02] and [DIGCOM01] are describing the impairments using mathematical models.

The output data of the channel decoder is, in case of no errors, exactly the same MPEG-2 transport stream as at the input of the channel encoder. The demultiplexer has now to choose - depending on the end-user input - which audio and video or data stream to demultiplex. The selected stream is then consumed by the end-user.

8 Interleaving is the scattering of information pseudo-randomly in the time or frequency domain.

9 A symbol in digital communication is the smallest amount of data transmitted at a time.

10 In a single frequency networks, different transmitters are synchronized and sending their data on the same radio frequency. This improves the coverage of the area and it allows seamless transition from one transmitter to another within mobile reception. 


\subsection{Software-defined radio}

The term software-defined radio or software radio was introduced in the 1990s to define an architecture of a transceiver which is mostly driven by software running on generic processors, rather than on dedicated hardware or signal processors.

"The [.. ] software radio revolution extends horizons by liberating radiobased devices from chronic dependency on hard-wired characteristics [...]. This liberation is accomplished through a combination of techniques that includes multi-band antennas and RF conversion; wideband [..] Analog/Digital/Analog conversion; and the implementation of IF, baseband, and bit-stream processing functions in general-purpose programmable processors." Software radio is running on "[..] programmable hardware, increasing flexibility via increased programmability." ([SRA95]) [SR02] defines the following properties of a software radio:

1) Its functionality is determined by software.

2) On its hardware, different standards can be executed, controlled by parameter lists.

3) All standards are looked at as members of one family.

In this work chapter 4 starting on page 32 falls into that category and describes one part of such an architecture.

As an example of a bigger software defined radio the Open Source project GNU Radio can be mentioned. It "is a free software toolkit for learning about, building and deploying software radios" initiated by Eric A. Blossom. ${ }^{11}$ It "provides the tools to start exploring" the radio frequency spectrum.

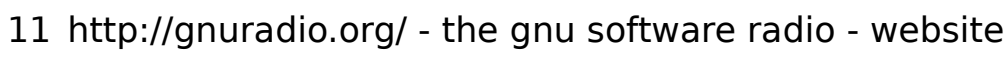




\section{Digital TV receiver front-ends}

The term front-end is an ambiguous term in the transceiver engineering world. Every block of a transceiver chain has a "front"-end and a "back"end.

For a digital TV receiver the term front-end describes typically the blocks between the antenna and the signal processors/decoder. This chapter will explain the blocks of a front-end by taking a DVB-T receiver as an example (Fig. 2.1).

DVB-T is the terrestrial digital TV broadcasting standard developed by the DVB project (see 1.2.1). DVB-T is using Coded Orthogonal Frequency-Division Multiplexing (COFDM). That modulation scheme is described elsewhere ${ }^{12}$, which is why it is not repeated here.

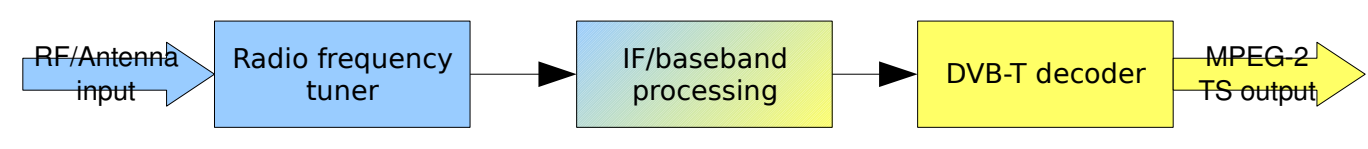

Fig. 2.1: Top-level block diagram of a DVB-T receiver

It shows the "classical" approach. It contains a tuner for down-converting the required radio frequency signal to the first intermediate frequency. $\mathrm{IF} /$ baseband processing is further filtering of the wanted signal and the conversion of the analog signal to its digital representation using an appropriate analog-to-digital converter. Finally the DVB-T decoder is demodulating and decoding the baseband signal to recover the MPEG-2 transport stream data. ([DVB05], p. 258f)

This top-level block diagram is not only applicable for digital TV receivers. In fact this architecture is common to all software-defined radios - even partial ones. ([SR02])

\subsection{Radio Frequency Tuner}

The tuner amplifies the analog RF signal, it is "tuning" a local oscillator in the way that the down converted product, the selected frequency, falls into the fixed (first) intermediate frequency (IF). Depending on the architecture 12 "Overview of the 3GPP Long Term Evolution Physical Layer", Freescale White paper about LTE, 2007 and in [DVB05] on page 237. 
of the receiver some tuners are converting the signal to a very lowintermediate frequency or even to zero-IF (baseband), sometimes there is more than one intermediate frequency before the wanted frequency is sampled by an ADC. The tuner can amplify the signal at each IF stage using optimized amplifiers for that particular range.

Fig. 2.2 shows a top-level block diagram of a RF tuner.

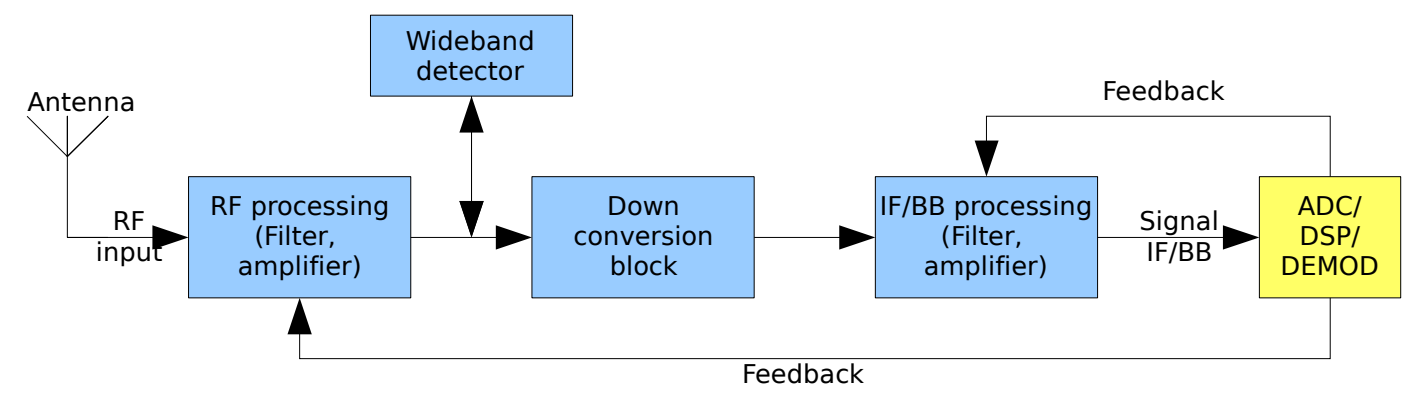

Fig. 2.2: RF tuner block diagram

As the losses of the down-conversion process cannot be gained back in later stages, it is the performance of the tuner at this level which significantly specifies the quality of the receiver. It is also at this very early stage in the receiver to take the necessary measures to remove or at least to reduce the impact of the interferences on the transmitted information.

Traditional tuners which were mainly designed for analog receivers do not work sufficiently well with DVB-T signals, mainly due to phase noise introduced by the local oscillator. The phase noise has almost no impact on analog TV signals, but it is critical for narrow band signals. A DVB-T signal is a constellation of many small-band carriers (8192 OFDM $1 \mathrm{kHz}$ carriers in $8 \mathrm{~K}$ mode) which makes it very sensitive for phase noise. It can also limit the maximum Signal-to-Noise Ratio of the whole receiver.

Another important characteristic for tuners is the noise figure. The noise figure gives the amount of additive noise introduced by the components used in the tuner. The noise figure should be as low as possible in order to improve the receiver's sensitivity. ([DVB05], p. 260f)

One other task of the RF tuner is to filter the IF-signal in order to reject adjacent channels which have been down converted along with the wanted channel (baseband filtering, image rejection). See 2.5.3 for more details as to why this is important. 
As the RF tuner is mixing and amplifying the signal, it introduces the results of non-linearities. Especially third-order intermodulation products are a problem. In 2.4 this problem is discussed.

The wideband detector is assisting the gain controller to make the right decisions. More discussions about that can be found in 3.2.2.

At the output of the tuner the IF signal has to be provided with a certain power, because as a next step, the signal is digitalized by an ADC. This ADC can only handle a signal in a defined power level range.

The digital representation of the analog signal is then processed by DVB-T decoder.

\subsection{DVB-T decoder}

In Fig. 2.3 a block diagram of a DVB-T receiver is shown. In this work only the two blue-colored (Front-end, Analog/Digital converter) are covered, but for completeness every block is drawn and mentioned. The yellow parts are the DVB-T decoder.

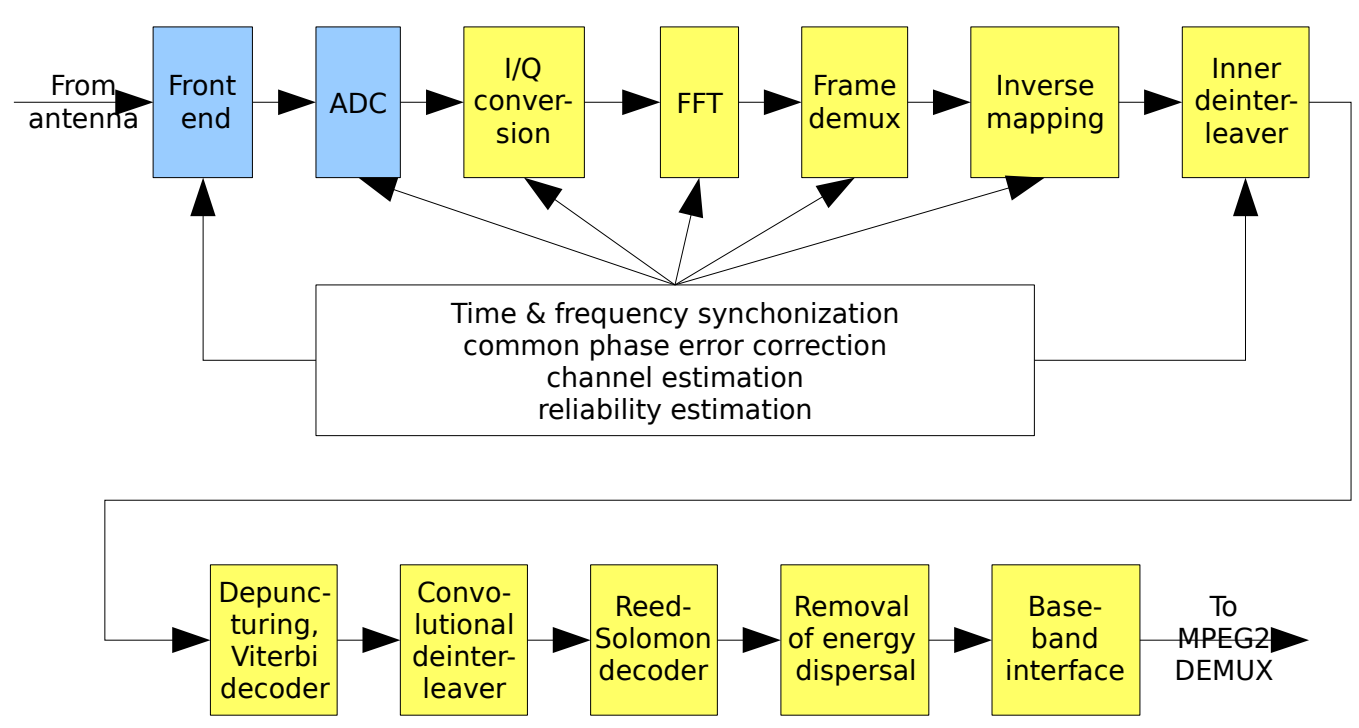

Fig. 2.3: Block diagram of a DVB-T receiver ([DVB05], p. 255)

The DVB-T standard EN 300744 does not specify the receiver side, but only the transmitted signal. The implementation of a receiver can be done in different manners and depends on the reception environment. 
The first DVB-T decoder hardware was consisting of three integrated circuits - the ADC, channel processing (COFDM demodulation) and channel decoding. Nowadays these blocks are integrated in one circuit; a very recent development has two RF tuner and two DVB-T decoders in one $8 \mathrm{~mm} \times 7 \mathrm{~mm}$ integrated circuit ${ }^{13}$.

A DVB-T receiver has to cope with several impairments that occur during terrestrial transmission (as described in 1.3). Additionally the front end inserts noise and phase noise (2.1).

Within the COFDM signal, pilot carriers are scattered in time and frequency with a known amplitude and phase. Those pilots are used to handle time and frequency synchronization and channel estimation. The channel estimation is giving the parameters to equalize each sub-carrier. Ideally this reverts the effects of the transmission channel. ([DVB05], p. 255ff)

In COFDM the data is modulated by changing the phase and the amplitude of a carrier. Each carrier has a certain frequency and there is a certain gap between carriers. As previously said, phase noise introduced by the tuner has a big impact on those narrow-band carriers in this modulation type. Additionally the oscillator which is providing the clock to the decoder IC can produce phase noise and must therefore be very accurate and stable. The IC should be also able to handle some inaccuracy of a oscillator.

\subsection{Interaction of decoder and tuner}

To have the signal at the input of ADC at the right power level, sophisticated receivers exchange information between the decoder and the tuner in order to control the amplifiers residing in the tuner.

The first DVB-T decoder circuits were not yet advanced enough, so that the control of the gain was done automatically inside the tuner. An internal feedback loop returned information about the signal power and the hardware-based control logic increased the gain when the signal level was too low and decreased the gain or attenuated when the signal level was too high, respectively. This is called Automatic Gain Control (AGC).

In order to solve this problem it was now the decoder to ask the tuner the

13 Press release of DiBcom's DiB29098: http://www.dibcom.info/lmages/Upload/pdf/ CP/PR_DVB-SH_MWC_080204_UK.pdf 
amount of gain needed to have the right signal input level. The digital TV decoder knows exactly at any time the input level, so it can request very accurately the right gain. Furthermore it has to be possible to calibrate the tuner's gain slopes and ranges to the decoder, so that it can apply the gain command properly.

The gain command is usually a voltage with is controlled within a certain range to set the desired amplification.

\subsection{Amplifiers}

As mentioned above the correct signal level is playing a very important role in a DVB-T receiver system. In order to have the right level the system must contain some amplifiers. As shown in Fig. 2.2, usually there are amplifiers at two or more places, one amplifier to add gain to the radio frequency stage (before down-conversion) and one (or more) to gain the signal at IF/baseband-stage(s).

Frequency amplifiers are sensitive analog components or circuits and depending on the technology used and the type and range of signal to be amplified the performance varies. The gain of an amplifier is given in decibel $(\mathrm{dB})$. The following properties and parameters are describing an amplifier ([HFT92], p. 5ff).

Stability: An amplifier needs to be stable. It has to change the amplitude of the incoming signal proportionally at its output. It has to amplify the signal and should not oscillate and thus not generate a signal on its own.

Efficiency: An amplifier is consuming the provided current and the power of the signal at his input to output the resulting modified signal. Additionally it loses current by creating heat, radiation and harmonic signals of the input.

Gain: The current consumed to increase the amplitude of the signal.

The ideal amplifier is linear, i. e. the gain increases proportional to the input power. In reality this is impossible, due to physical limitations. This is why the following characteristics are covered under the term Nonlinearity. ([HFT92], p. F 27ff).

1-dB gain compression point: Comparing the output power and the input power (in $\mathrm{dB}$ ) relation with a curve, one will get the chart Fig. 2.4. Increasing 
the input power the most important component - the transistor - is saturating. The point where the real output power is $1 \mathrm{~dB}$ lower than it would be in a linear range is called 1-dB gain compression point.

Harmonics: Because of the nonlinearity the amplifier is creating harmonic

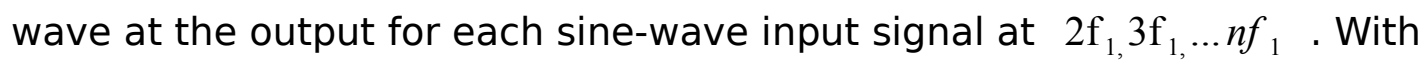
growing $n$ the amplitude of the harmonic is smaller and smaller - increasing the gain of the amplifier is increasing the amplitude of the harmonics linearly. Usually a filter at the output is removing harmonics, which are not in the wanted frequency range.

Intermodulation: Intermodulation is the interaction between neighboring frequencies in the input signal. Depending on the distance between those signals the second order intermodulation product $\left(\mathrm{IM}_{2}\right)$ is not a problem, because it does not fall inside the wanted frequency range.

$$
f_{2} \pm f_{1}
$$

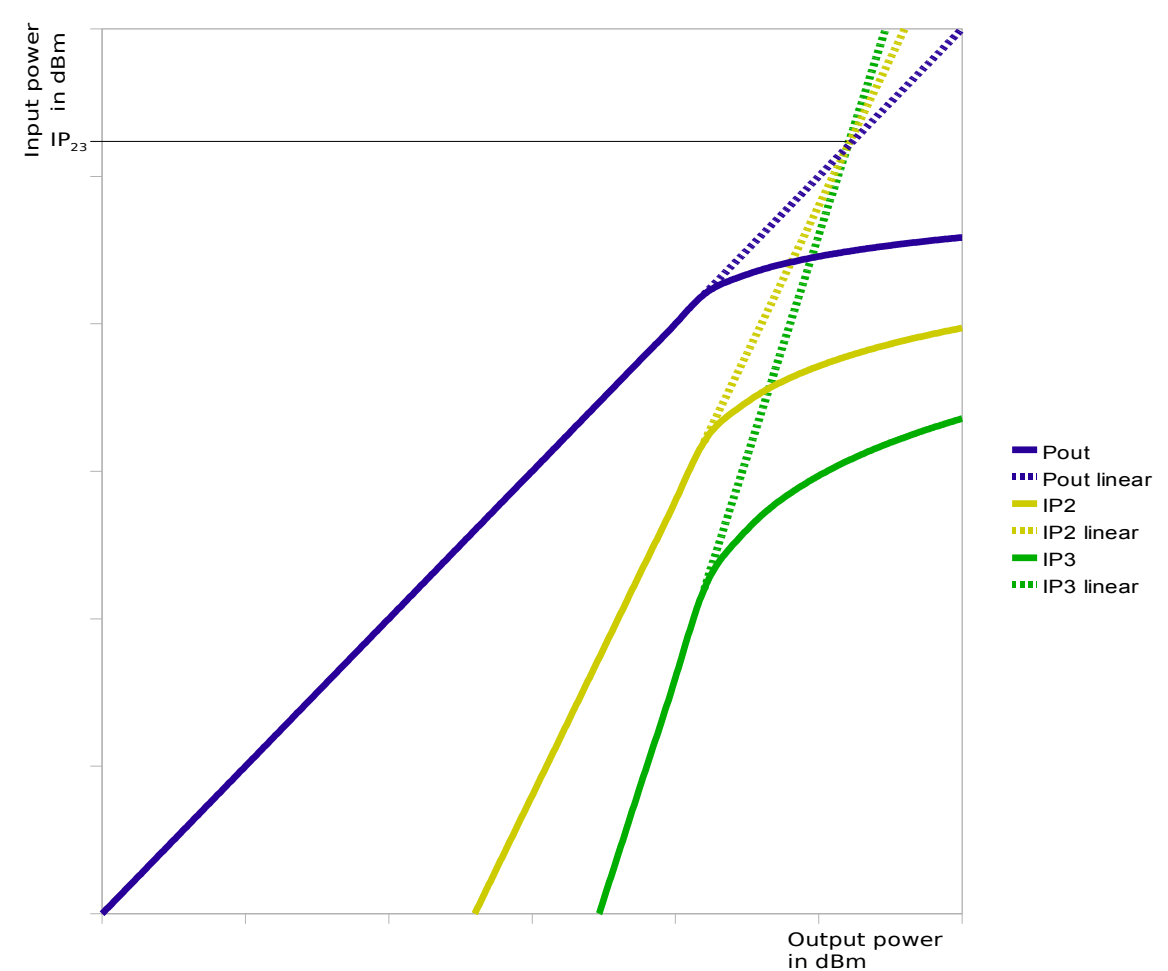

Fig. 2.4: Amplifier output vs. input power in $d B$ - showing the third order interception point.

The power of the second order IM is growing two times faster than the wanted signal. For example increasing the gain of the wanted signal by $2 \mathrm{~dB}$ the power if the $\mathrm{IM}_{2}$ is growing by $4 \mathrm{~dB}$

For the $\mathrm{IM}_{3}$ the power is growing by factor three compared to the wanted 
signal. Additionally the third order intermodulation product almost always falls into the wanted range.

$$
2 \cdot f_{1}-f_{2}, 2 \cdot f_{2}-f_{1}
$$

Fig. 2.4 shows the power curves of the signal and its intermodulation products. It also visualizes the nonlinearity problematic and the third-orderinterception point, which is given for each amplifier as a quality indicators. A special case of intermodulation is crossmodulation in which neighbored signals of different modulations are modifying each other.

\subsection{Analog to digital conversion}

Is the signal to be processed an analog one, it has to be digitalized with an analog-to-digital converter (ADC) before it can be processed by a DSP. The nature of an $A D C$ is to put out a numerical value describing the state of the input signal at a certain moment in time within a certain range. In other words, converting a continuous signal into a discrete signal.

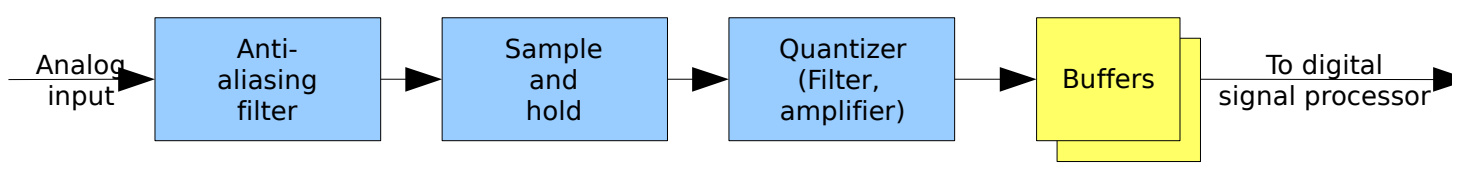

Fig. 2.5: Elements of ADCs used for RF and IF digitization

The AD-conversion is done in three steps ([DIGNT02] p. 27f): sampling, quantification and coding. The bandwidth (B) and the amplitude of the signal to be sampled must be defined and limited.

\subsubsection{Sampling}

Sampling is recording the values of the signal at a certain moment with a defined period $T_{s}$. The inversion of this period is the sampling frequency $f_{s}$.

$$
T_{s}=\frac{1}{f_{s}}
$$

According to Nyquist and Shanon ([DIGNT02] p. 119ff) the relation between the bandwidth of the signal and the sampling frequency is as follows:

$$
f_{S} \geqslant 2 \cdot B .
$$

Is this rule not followed, the recorded values of the signal are not enough to 
give the right image of the signal. In practice, especially when the bandwidth is relatively high, not only a sampling frequency equal two times the bandwidth is applied, rather a higher sampling bandwidth is chosen to be able to digitally filter the signal.

\subsubsection{Quantification and coding}

When doing the quantification, the amplitude range of the input signal is divided into a finite number of intervals. Each interval has its number. Like that the value recorded by the sampling process, which can be of infinite precision, is filled into one of the intervals. The data width of those intervals is representing the precision of the digitalization process.

The coded output of an ADC are binary numbers, hence the resolution of the precision is done using the unit bit. Each additional bit available doubles the precision of the ADC.

Is the precision too low the quantification error or quantification noise is high. This is unavoidable in all types of ADCs. Depending on the expected power variation of the input signal an ADC has to have a certain precision to avoid quantification noise.

\subsubsection{Out-of-band energy}

When sampling a signal at IF stage using the Shanon/Nyquist-rate it is assumed that the signal in band-limit. A theoretically defined band-limited signal is a signal with no frequency components above a certain frequency. Real radio signals at the input of a receiver always have signals with higher frequencies present.

It is a matter of the relative amplitude of these undesired frequencies (above one half the sampling rate) to the desired ones, thus the relative power, that is important. It can create a spectrum overlap and distort the desired signal. An example is shown in Fig. 2.6. a) illustrates the spectrum with the desired component and the undesired one. Sampling the desired signal at two times the highest frequency result in the spectrum b). This is where the overlap occurs. The undesired signal is inside the desired one. 


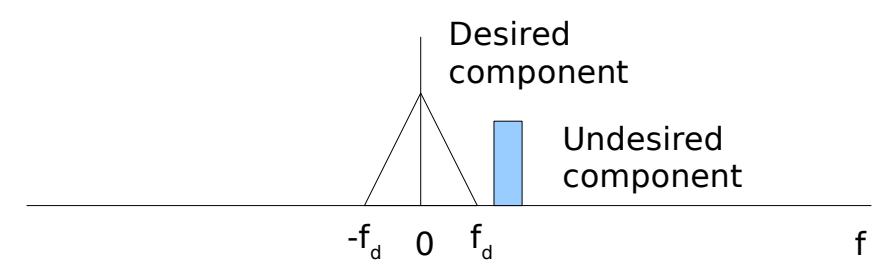

a)

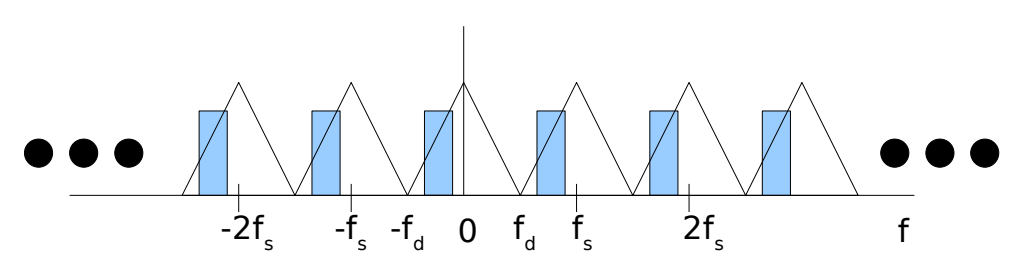

b)

Fig. 2.6: Spectrum of: a) a signal with a desired and undesired component and $b$ ) the signal sampled at $f_{s}=2 f_{d}$. ([ADCRF95])

Depending on the power of the undesired signal it can cause severe distortions in the reconstruction and processing of the desired signal.

Distortion due to spectrum overlap can dominate distortion created by ADC nonlinearities when undesired signals appear within the Nyquist band (DC to one-half the sampling rate).

In case of a receiver the RF tuner has to provide appropriate filters to avoid strong undesired signals in the digitized spectrum. A performance criterion has to be used to evaluate the quality of the reception of the desired signal with having a undesired signal present. ([ADCRF95])

\subsection{RF performance specifications}

In the full frequency range of a terrestrial transmission system, a lot of channels are allocated for various applications. An adjacent channel in is defined as a AM, FM or another TV signal which can impair the reception of the desired signal due to its power and proximity. These adjacent channels are adding noise power in the spectrum.

The $\left[\text { MBRAI07] }{ }^{14} \text { and partially the [NORDIG07 }\right]^{15}$ receiver performance

14 MOBILE AND PORTABLE DVB-T/H RADIO ACCESS Interface specification written by the European Information, Communications and Consumer Electronics Technology Industry Associations

15 NorDig is specifying a common platform for Digital Television to be used within the Nordic region (Denmark, Finland, Iceland, Norway and Sweden), but 
specifications define, among others, how a DVB-T/H broadcast receiver should be resistant to either analogue or digital interferers. With the given immunity figures, it is possible to evaluate the capacity of a receiver to accept the presence of powerful interferers. This immunity figure represents the receiver's capacity to make difference between the useful signal and all others: The Interferer Selectivity. (Fig. 2.8 and Fig. 2.7, see also section 2.5.3)

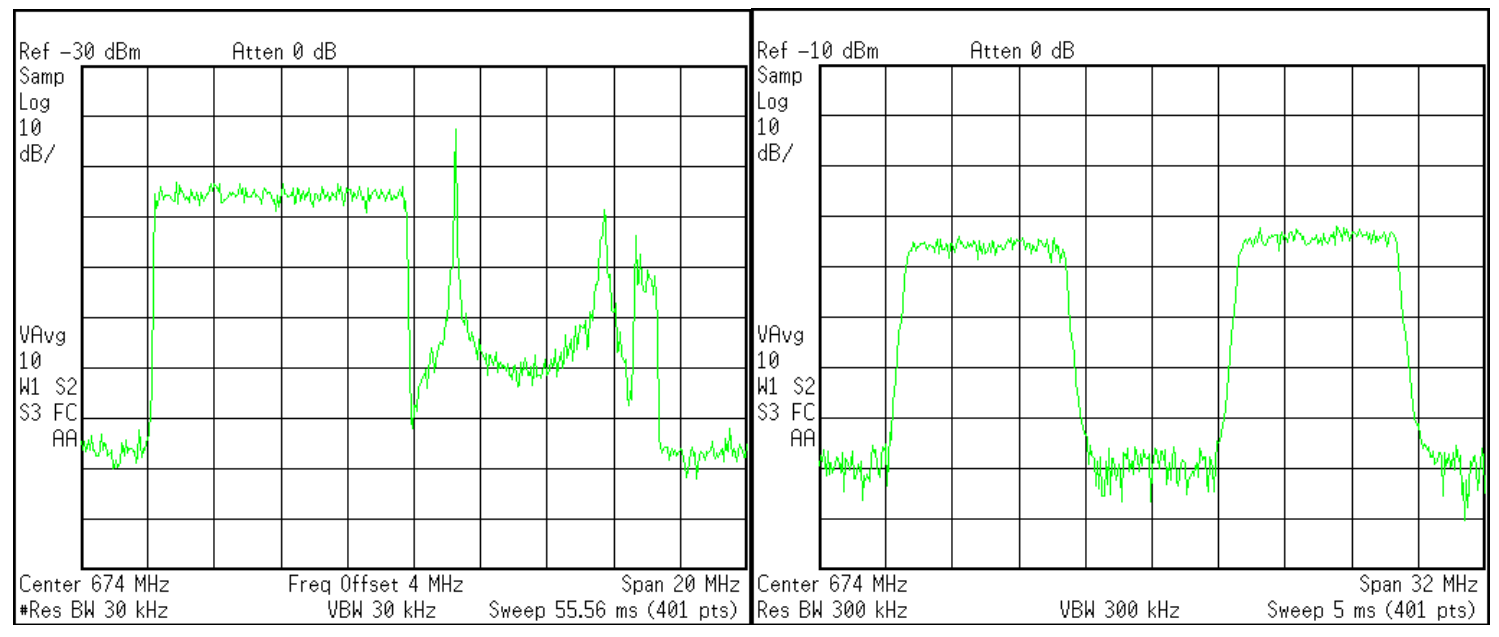

Fig. 2.8: Frequency spectrum of a $N 1$ adjacent

Fig. 2.7: Frequency spectrum of a $\mathrm{N1}$ channel pattern (Analog) next to the wanted adjacent channel pattern (Digital) next to the digital signal $(N+1)$ wanted digital signal $(N+2)$

Measuring this immunity figure with a single adjacent channel does not represent very accurately real conditions where several interfering signals are present. Therefore in addition to the single interferer approach, multiple interferers configuration are proposed. Double analogue (Fig. 2.10), digital and mixed (Fig. 2.9) signals are introduced to know the immunity of the receiver for third order intermodulation (IP3). (see also section 2.4) In such a scenario the number of interfering signals has been limited to two.

As the mentioned specifications are generally a question of RF performance rather than digital demodulation ability, at DiBcom these scenarios have been adopted to other digital TV standard such as ISDB-T and DAB, because the front-end architecture is similar between the different digital systems. 


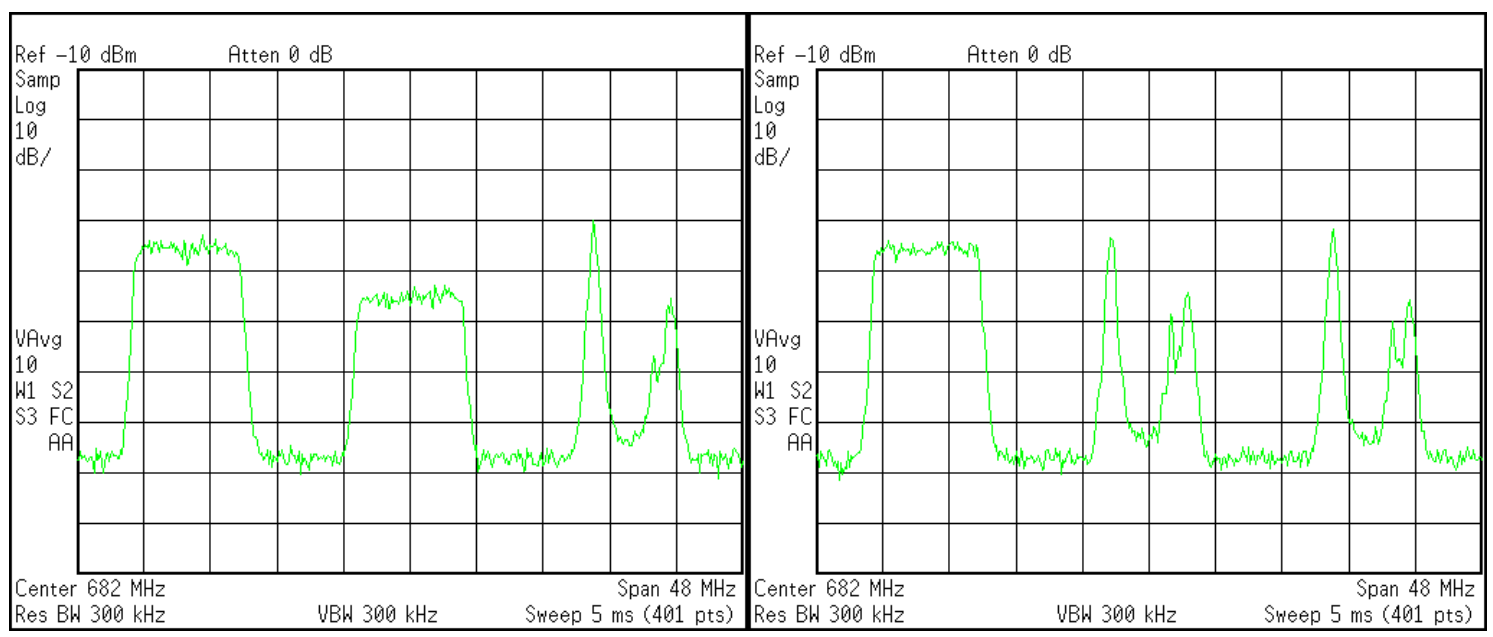

Fig. 2.9: Frequency spectrum of a $L 1$ adjacent

Fig. 2.10: Frequency spectrum of a $L 2$ channel pattern (Digital/Analog mixed) and the desired digital channel (left) adjacent channel pattern (double analog) and the desired digital channel (left) 


\section{DiBcom front-end architecture}

\subsection{Historical development at DiBcom}

The front-end architecture of DiBcom has not evolved a lot before August 2006; the classical approach, described in 2 was followed. At that time DiBcom announced the availability of new integrated circuits, intended to simplify DVB-T and DVB-H digital reception on all mobile and portable devices. Later other digital TV standards followed using the same new technology.

\subsubsection{In-can RF tuners - before 2005}

Up to the year 2004 at DiBcom the the front-end was composed of an RF tuner, commonly called "in-can" as it is housed in a metallic casing, a Surface Acoustic Wave filter (SAW), optionally an IF amplifier, a crystal and a DVB-T demodulator in baseband.

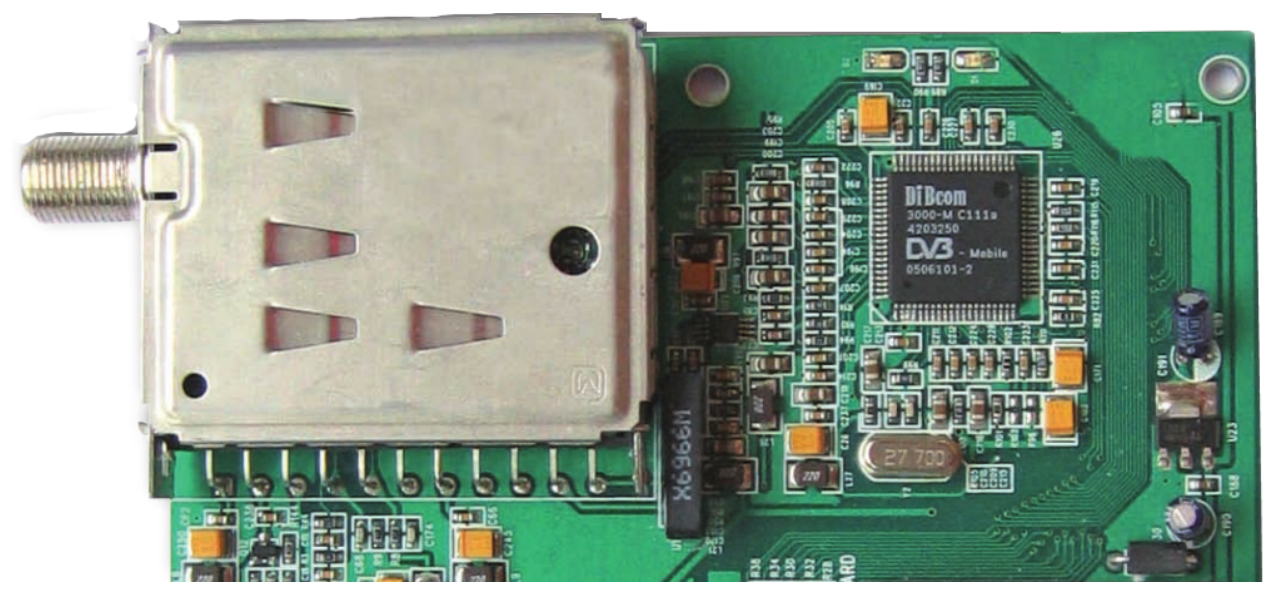

Fig. 3.1: A classic DVB-T front-end using an in-can RF tuner and a silicon demodulator (before 2005)

This bulky implementation was very large: the in-can RF tuner measures $4 \times 5 \times 1 \mathrm{~cm}$. In addition, this tuner type is consuming a high level of power (approximately $700 \mathrm{~mW}$ ) and requires a great deal of fine-tuning before leaving the factory. The radio frequency filters need to be centered on the wanted channel, and therefore must be able to track the desired frequency when the system selects a given channel. This characteristic makes it difficult to integrate, and it is usually implemented with discrete 
components such as coils and variable capacitors.

This results in a dispersion of the receiver's characteristics (sensitivity, filtering, etc).

Fig. 3.1 shows a picture of a classic DiBcom-based DVB-T front-end having an in-can RF tuner and a DiB3000 DVB-T demodulator.

\subsubsection{Silicon RF tuners - 2005}

Further advances in silicon technology lead to one-chip tuners, which have become available in 2005. These no longer require the integration of a "classical" tuner module into the receiver, but consist of a single chip which accepts the RF signal on one input, and outputs the first IF signal or later "Zero-IF". Only a minimum of external passive components are required.

The advantages of this concept are: Tuners are small, light-weight and can be integrated in the receiver easily. Of course it is also a question of cost in production. Disadvantages are for example that the power dissipation is higher, the performance is slightly lower and the cost of the component is higher. ([DVB05] p. 266f)

Fig. 3.2a shows an example of DiB3000 with Microtune's silicon tuner MT2060.

\subsubsection{System in Package (SiP) - 2006}

In August 2006 DiBcom announced the availability of the DiB7070 family. This integrated circuit utilizes the System-in-Package (SiP) technology to integrate two dies (RF tuner and demodulator) in one chip. This approach offers real advantages to digital television applications in terms of performance, size and cost reduction. The high level of integration has proven to be able to replace the previously used front-end-types.

Fig. 3.2b shows an example of DiB7070. The dimensions are $12 \times 12 \mathrm{~mm}$. The integration of a silicon RF tuner and a demodulator in the same component helps to solve the problems of space, characteristics dispersion and cost.

The tuner/demodulator coupling has a big impact on gain control optimization and prevents the duplication of modules in both circuits. Moreover, the integration is especially efficient when a "Zero IF"-tuner 
output is used because the expensive external RF SAW filter is replaced by a simple digital filter in the demodulator. A simple crystal oscillator is therefore the only external component required.
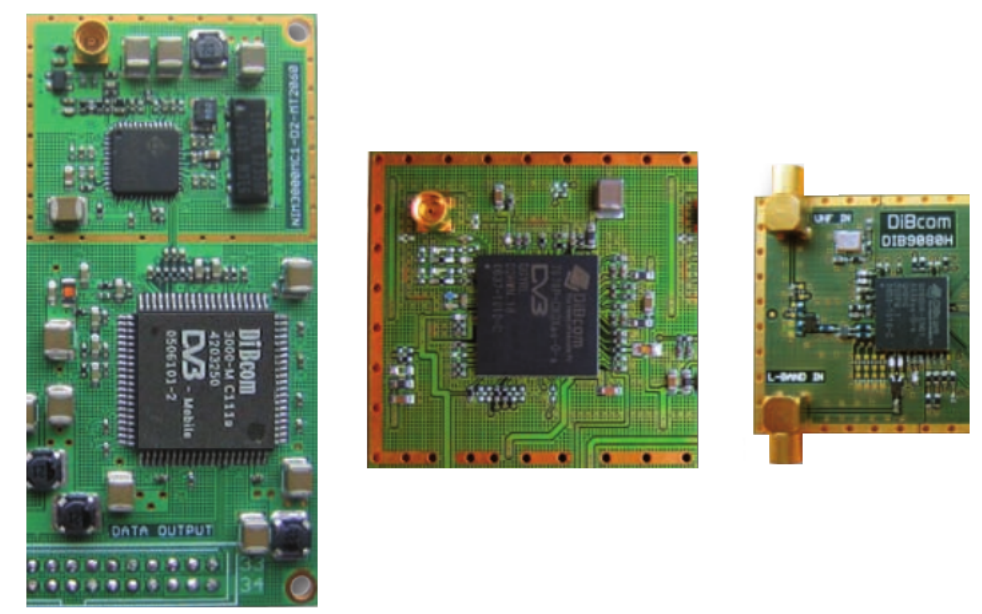

Fig. 3.2: a) Two chip front-end; $R F$ tuner and demodulator (2005, left); b) System in Package - One chip front-end. RF tuner and demodulator in one package. (DiB7070,

$12 \times 12 \mathrm{~mm}, 2006$, center); c) System in Package - smaller package (DiB9080, 7x7mm, 2007, right)

The power consumption is about $400 \mathrm{~mW}$ in DVB-T mode.

In late 2007 the DiB8070 was introduced using the SiP-technology to create a single-chip ISDB-T receiver.

\subsubsection{System in Package (SiP) - today}

With the introduction of $90 \mathrm{~nm}$ technology at DiBcom the decision was made to put the $A D C$ into the tuner-die in order to safe space on the demodulatordie. The reason for that was to rearrange the dimensions of the complete chip to create an even smaller package.

The first product using that technology was the DiB9080 (Fig. 3.2c). This $7 \times 7$ $\mathrm{mm}$ package is only $40 \%$ of the space of a DiB7070 by providing the same functionality. Additionally the power consumption of a $90 \mathrm{~nm}$-based device is lower than other (bigger) technologies.

Because of that architecture change the traditional AGC mechanisms were not usable any longer and a new way had to be developed. 


\subsection{Traditional Automatic Gain Control}

The Automatic Gain Control mechanism developed originally for the "in-can" tuners and was later used for silicon RF tuners and also the first generation SiPs is now called classical or traditional.

In this traditional AGC the demodulator handles simultaneously baseband and RF amplifiers. Two gain commands (AGC1 and AGC2) are used for that purpose. Both of them are generated from a common (or global) command given by the AGC loop.

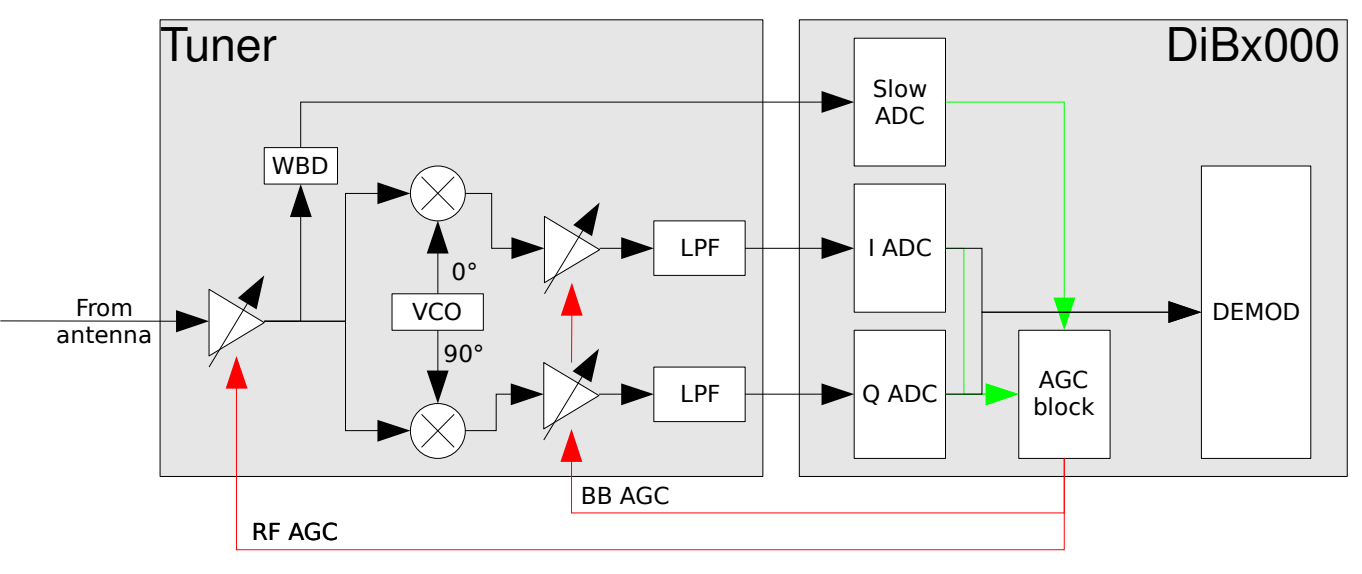

Fig. 3.3: DiBcom's traditional front-end architecture highlighting the traditional AGC loop (red and green)

The AGC loop offers high input dynamic range control with the help of the compensation of the amplifiers (baseband and RF) with voltage control gain responses. This allows the overall gain (Gain $(\mathrm{dB})$ ) to be a linear function of the common AGC. It can detect the presence of an analog adjacent channel and can change the target $A D C$ power in order to optimize the input level without saturation.

\subsubsection{Signal power}

The signal power is given by the ADC. It is the primary indicator to figure out, how much gain is required. It is compared with the configured ADC target level and the gain is adjusted appropriately: Is the level too high, the gain is reduced, is it low, the gain is increased.

As the feedback of the adjusted gain immediately is seen by the ADC the 
gain accuracy is not so important.

\subsubsection{Wideband power}

A dedicated loop is used to split the overall gain on RF and BB amplifiers. For that purpose each amplifier ( $R F \& B B$ ) response can be modeled by four successive linear curves as shown in Fig. 3.4.

The RF command is set to avoid saturation of the RF amplifier due to the strengths of neighboring channels to reduce the effect of non-linearities. The gain-split between RF and BB amplifiers therefore takes into account the value of the RF wide band detector. This split is also called take-over-point because at this point in the global AGC command the baseband amplifier is taking over some gain which normally should have been applied using the RF amplifier.

Only newer tuners provide that device. It is a component which is positioned close to the RF-processing block (see Fig. 2.1) and it gives the power level of a complete frequency band. ([DCRDVBH05])

\subsubsection{Gain ranges and slope configuration}

Each of the values given the in the chart in Fig. 3.4 can be programmed to the hardware to enable optimal gain control depending on the tuner amplifier's gain response.

In Fig. 3.4 AGC1 is used for the RF amplifier and AGC2 for the baseband amplifier. From the top view the AGC block gives a global AGC command. It represents a point on the total gain curve which corresponds a certain applied gain. 


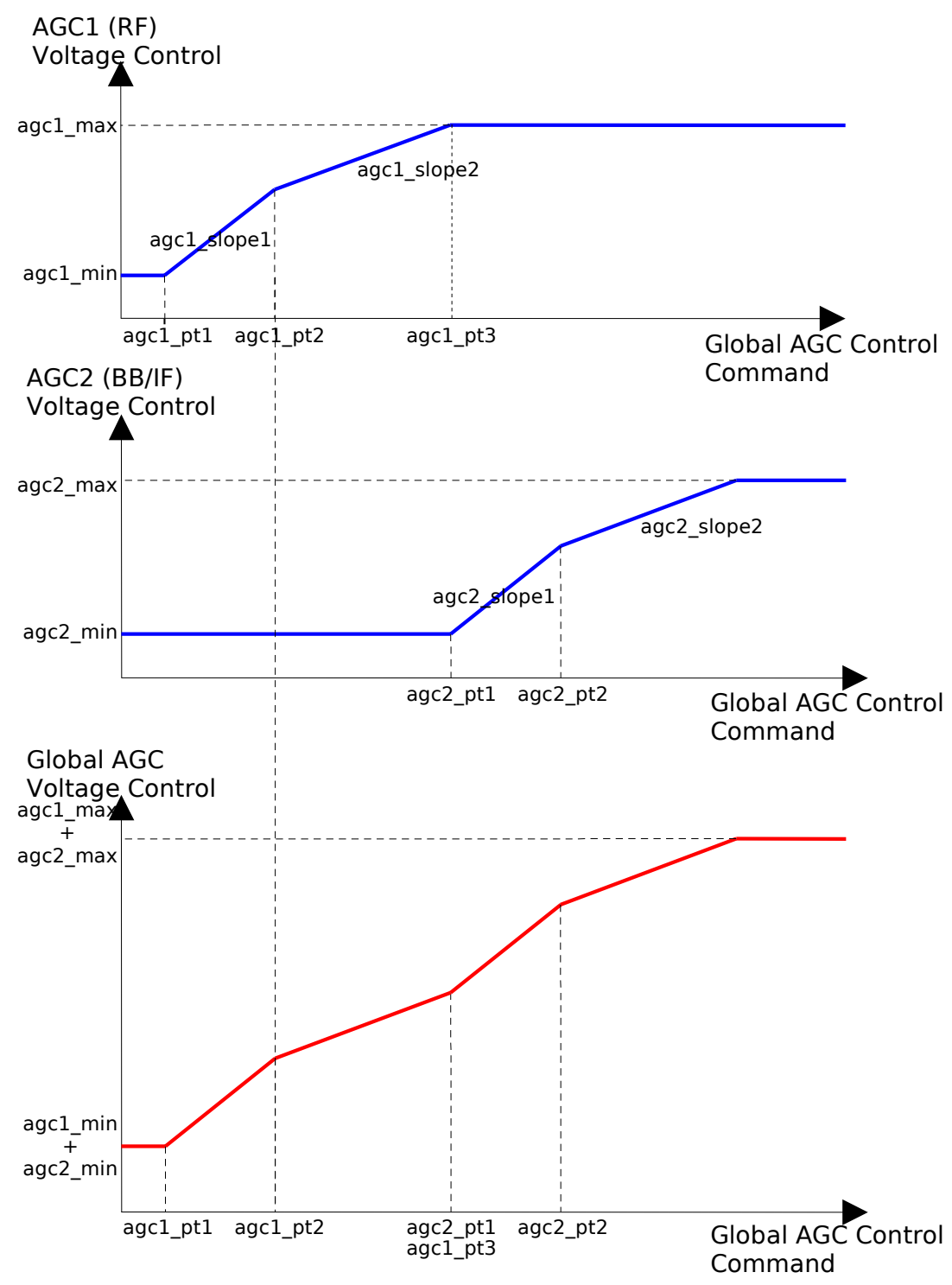

Fig. 3.4: Configuration of a traditional AGC hardware block

\subsubsection{Convergence speed and optimal ADC-target}

The delay the AGC introduces to react on a signal level change is critical, because it can significantly reduce the recovery time of the channel decoder.

Very common when receiving under mobile or portable conditions are the sudden drops or raises of the signal level. Due to buildings or tunnels a direct view to the transmitter can be hidden and the signal level can suddenly get very low or high. The gain control loop here has to adapt quickly enough to reduce the time the $A D C$ is only seeing noise or is 
saturated respectively.

The contrary is required in mobile conditions, where a slow or even better no convergence would be good: Depending on the moving speed of the receiver relative to the transmitter the signal is affected by dynamic fadings which lead to a strongly varying signal power level at the ADC. As the task of the gain control loop is to compensate for signal level variations and to keep the power level at the ADC constant at a certain target, here it is important that the variations are not flattened. All that in order to let the signal processing block select the correct algorithms to handle a mobile channel ${ }^{16}$. The ADC-target-power must be adjusted wisely, to not saturate the signal when having the ADC-target too high or to not put the moving signal-power below the noise when having the ADC-target-power to low. Is the transmitter or the receiver moving relatively to its counter-part the Doppler effect occurs. For more information about the Doppler effect in Digital TV systems see [DOPPLER07].

\subsection{Next generation front-end architecture}

With the introduction of the DiB9080 (Fig. 3.2c), the architecture of the front-end has changed significantly. Main differences are that the ADC has been moved to into the RF tuner and the AGC loop block has been completely removed from the demodulator.

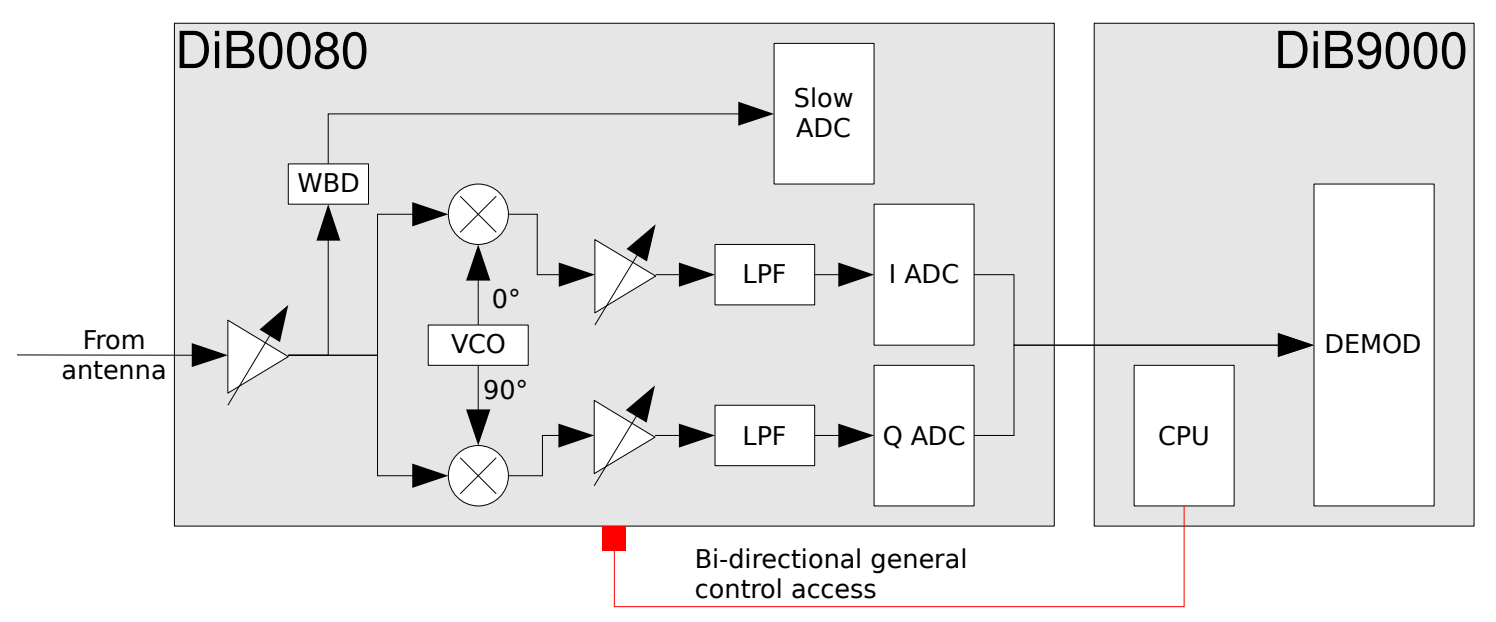

Fig. 3.5: DiBcom's new front-end architecture; A generic CPU controls the tuner.

Instead of that hardware block, now a generic CPU is in charge of controlling

16 The actions taken by the channel processing block to handle mobile channels depend on the transmission standard and the coding used. 
the amplifiers through the hardware registers of the tuner (red line in Fig. 3.5). The following chapter describes the process of development and implementation of that software-based gain control loop in detail. 


\section{Appendix}

\section{A Bibliography}

DIGNT02: Lochmann, Dietmar, Digitale Nachrichtentechnik, 2002

DIGCOM01: Proakis, John G., Digital Communications, 2001

DVB05: Reimers, Ulrich, DVB, 2005

WSIGNAL: Wikipedia, Wikipedia - Signal (electrical engineering), 2008, http://en.wikipedia.org/wiki/Signal_(electrical_engineering)

KNM07: Eylert, Bernd; Eylert, Dorothee, Kompendium Numerische Mathematik, 2007

WDAB: Wikipedia, Wikipedia - Digital Audio Broadcasting, 2008, http://en.wikipedia.org/wiki/Digital_Audio_Broadcasting MBRAI07: EICTA, MOBILE AND PORTABLE DVB-T/H RADIO ACCESS, 2007 SRA95: Mitola, Joe, The Software Radio Architecture, IEEE Communications Magazine, p. 26-38, No. 5, 1995

SR02: Wiesler, Anne; K. Jondral, Friedrich, A Software Radio for Second- and Third-Generation Mobile Systems, IEEE TRANSACTIONS ON VEHICULAR TECHNOLOGY, p. 738-748, No. 4, 2002

HFT92: K. Lange, K.-H. Löcherer (Hrsg.), Hochfrequenztechnik, 1992 ADCRF95: WEPMAN, JEFFERY A., Analog-to-Digital Converters and Their Applications in Radio Receivers, IEEE Communications Magazine, p. 39-45, No. 5, 1995

NORDIG07: NorDig, NorDig Unified Test specification, ver 1.0.3, 2007 DCRDVBH05: Antoine, P. et al, A Direct-Conversion Receiver for DVB-H, ISSCC, WIRELESS RECEIVERS FOR CONSUMER ELECT., p. 426-428, No. , 2005 DOPPLER07: Sobol, Michael, The Doppler Effect and Digital Video Broadcasting, 2007

SAGC: Kim, Chonghoon; Im, Sungbin, Digital automatic gain control for software radio W-CDMA base stations, IEEE ELECTRONICS LETTERS, $p$. 318-320, No. 3, 2002 


\section{B Examples for signal in frequency and time domain}

In Fig. 6.1 a time-domain representation of signal is shown. From this view it is very hard to see of which components it consists. Only the plot of this signal in frequency-domain (Fig. 6.2) gives a conclusion. This signal is built of two waves, one which has a frequency of $1 \mathrm{MHz}$ and one which is $2 \mathrm{MHz}$. Seeing the time-domain representation is reality will show, that the two sinusoids are uncorrelated.

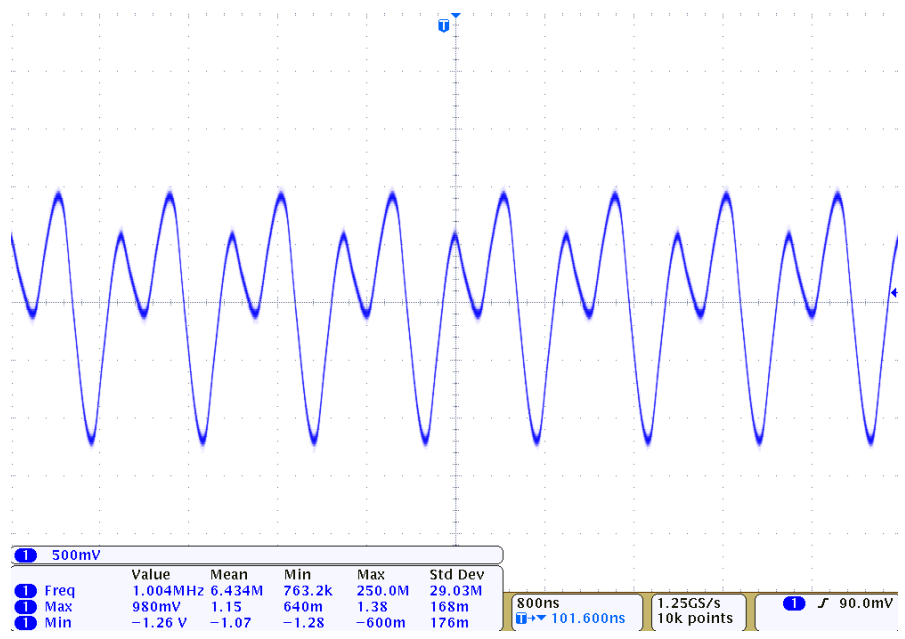

Fig. 6.1: A signal with uncorrelated 1 and $2 \mathrm{MHz}$ sine waves in time-domain representation

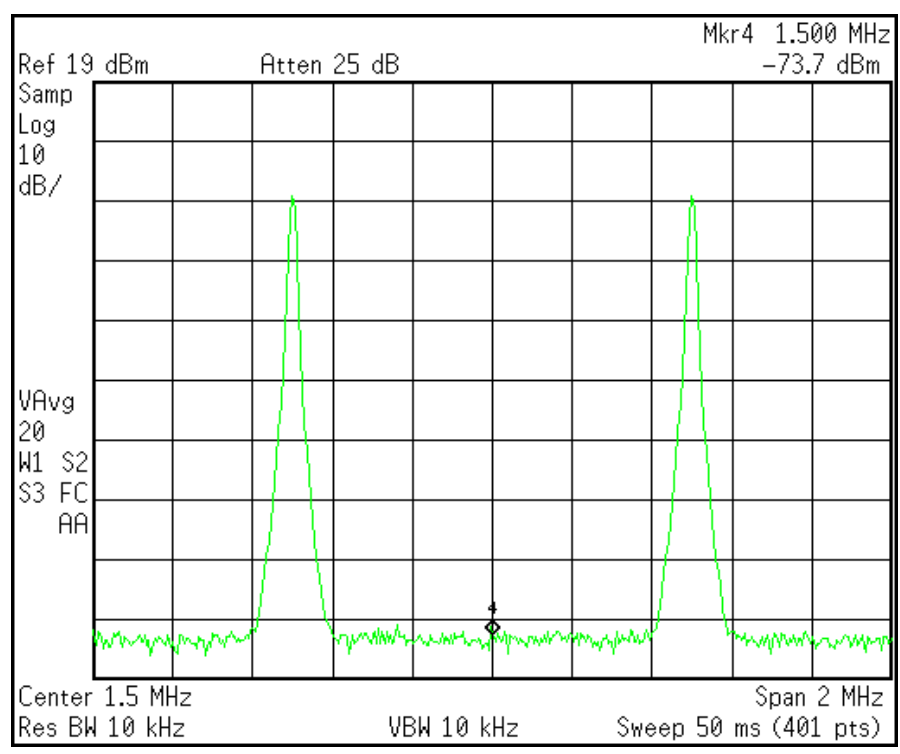

Fig. 6.2: A signal with unsynchronized 1 and $2 \mathrm{MHz}$ sine waves in frequency-domain representation

It gets even more difficult to watch the time-domain representation when it comes to more complex signals. In Fig. 6.3 the oscilloscope is overburdened with showing useful information about the signal. Again the representation 
in frequency domain is giving a clue: it is a Gaussian channel with a bandwidth of $7 \mathrm{MHz}$ centered at $10 \mathrm{MHz}$.

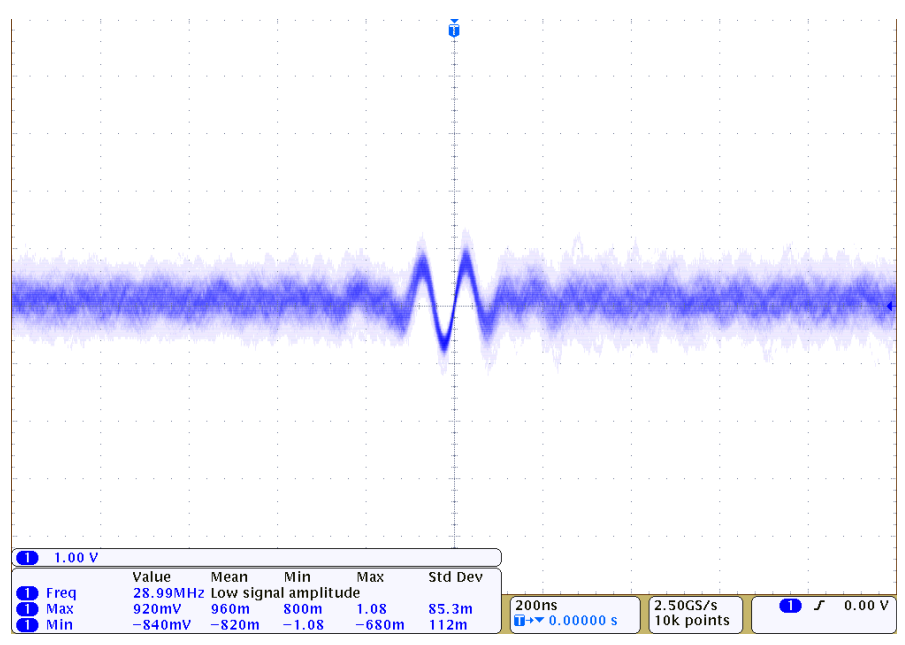

Fig. 6.3: $7 \mathrm{MHz}$ DVB-T signal (modulated on $10 \mathrm{MHz}$ ) in time-domain

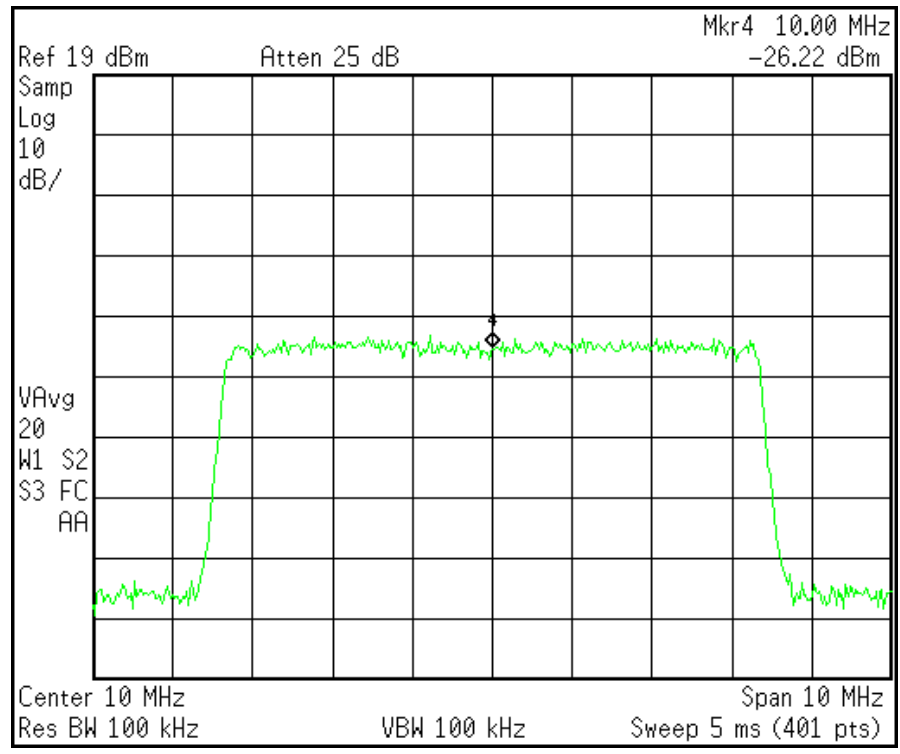

Fig. 6.4: $7 \mathrm{MHz}$ DVB-T signal (modulated on $10 \mathrm{MHz}$ ) in frequency-domain 


\section{E Selbständigkeitserklärung}

Hiermit versichere ich, dass ich die vorliegende Diplomarbeit selbständig und unter Verwendung der angegebenen Quellen und Hilfsmittel verfasst habe.

Die Arbeit wurde bisher in gleicher oder ähnlicher Form keiner anderen Prüfungsbehörde vorgelegt.

Antony, den 10. März 2008 